\title{
Enhanced Tumour Growth and Impaired Cellular Antitumoural Defence in Hepatic Colorectal Carcinoma Metastasis in Rats after Laparoscopy Compared to Open Surgery
}

\author{
D. Th. Inderbitzin, MD, ${ }^{1}$ G. R. Marti, ${ }^{2}$ S. Eichenberger, ${ }^{2}$ H.- M. Hoogewoud, MD, ${ }^{3}$ \\ L. Kraehenbuehl, $\mathrm{MD}^{1,4}$

\begin{abstract}
${ }^{1}$ Department of General Surgery, Hôpital Cantonal Fribourg, CH-1708, Fribourg, Switzerland
${ }^{2}$ Department of Histology and Embryology, Faculty of Medicine, University of Fribourg, CH-1700, Fribourg, Switzerland

${ }^{3}$ Department of Radiology, Hôpital Cantonal Fribourg, CH-1708, Fribourg, Switzerland
\end{abstract} \\ ${ }^{4}$ Department of Visceral Surgery, Lindenhof Spital, Bremgartenstrasse 119, CH-3012, Berne, Switzerland
}

\begin{abstract}
Background: This study aims to assess postoperative hepatic growth of colorectal adenocarcinoma metastasis and peritumoural macrophage counts after laparoscopy in an experimental animal model.

Methods: Thirty male syngenic $\mathrm{WAG} / \mathrm{Rij}$ rats were randomised into two surgical groups: laparoscopy (LS; $n=15$ ) using $\mathrm{CO}_{2}$ at $12 \mathrm{mmHg}$ and laparotomy (LT; $n=15$; negative control) during an operating time of $90 \mathrm{~min}$. At $45 \mathrm{~min}$ after setup, CC531s colon adenocarcinoma cells were injected into two liver lobes. Postoperative tumour volumes were determined by abdominal magnetic resonance imaging (MRI) and computed three-dimensional volumetry. Peritumoural macrophages were counted by local stereology using a confocal laser-scanning fluorescence microscope.

Results: The median postoperative tumour volume was significantly higher after LS in both lobes (L): after 10, 15 and 20 days in L2 and L5: 24/12, 54/38, 275/62 $\mathrm{mm}^{3}$ and $0 / 0,15 / 11,55 /$ $24 \mathrm{~mm}^{3}$ (LS/LT). Significantly fewer peritumoural macrophages were found after LS at all postoperative time points (Mann-Whitney: $p<0.05$ ).

Conclusions: Increased hepatic growth of colorectal adenocarcinoma metastasis and impaired cellular antitumoural defence after LS cast doubt on the use of LS in colorectal cancer and needs further clinical investigation.

Key Words: Colorectal carcinoma-Hepatic metastasis - Laparoscopy-Tumor growth-Antitumoral immunological defense.
\end{abstract}

Laparoscopic colic or rectal resection as part of the treatment for colorectal cancer has been adopted due to several advantages over laparotomy, such as

Data have been presented at the 1st World Congress of Endoscopic Surgery, EAES, Berlin 2006.

Address correspondence and reprint requests to: L. Kraehenbuehl, MD; E-mail: lukas.kraehenbuehl@bluewin.ch shorter hospital stay, less postoperative pain and faster convalescence. ${ }^{1-3}$

However, the influence of laparoscopy on intrahepatic colorectal tumour growth and antitumoural immunological defence is still under debate. Large clinical studies ${ }^{4-6}$ are still ongoing to establish the clinical relevance of laparoscopy in colorectal cancer treatment, but have not distinguished any significant impact on the outcome (5-year survival rate) compared to laparotomy. A couple of experimental 
studies have assessed the influence of laparoscopy on postoperative outcome of colorectal carcinoma using various models. The assessment of haematogeneous hepatic tumour spread in rats ${ }^{7,8}$ and mice ${ }^{9}$ and of renal or cutaneous tumour growth after subcapsu$\operatorname{lar}^{10}$ or subcutaneous ${ }^{11}$ injection of colorectal carcinoma cells have led to controversial for and against arguments for the laparoscopic technique. Importantly, hepatic macrophages have been described as playing a key oncological role in cellular antitumoural defence in respect of enhanced experimental tumour growth following selective depletion of phagocytotic cells. ${ }^{12,13}$ The influence of laparoscopy on the number and function of macrophages is not yet clear, although a lack of phagocytotic activity during laparoscopy ${ }^{14}$ has been described and semiquantitative measurements of hepatic macrophages in experimental colorectal hepatic metastases without significant difference between laparoscopy and laparotomy have been performed. ${ }^{8}$

Therefore, this study aims to assess the impact of experimental laparoscopy on postoperative hepatic growth of colorectal adenocarcinoma metastases and numbers of peritumoural macrophages assessed by MRI volumetry and immunofluorescent microscopic morphometry in a small-animal model.

\section{MATERIAL AND METHODS}

\begin{abstract}
Animals
Thirty male WAG/Rij rats (a Wistar-derived strain), weighing 290-320 g (Harlan, Horst, The Netherlands), were used for all the experiments. They were kept under specific pathogen-free (SPF) laboratory conditions with a controlled climate and light cycle and were fed a standard rat chow (Kliba Futter, Basel, Switzerland) with sterile water ad libitum before surgery. The animals were given 2 weeks of acclimatisation time and fasted $6 \mathrm{~h}$ before surgical intervention. The experiments were approved by the animal ethics committee of the State of Fribourg and performed according to international guidelines.
\end{abstract}

\section{Tumour Cell Line}

The CC531s cell line is a 1,2-dimethylhydrazineinduced, moderately differentiated colon adenocarcinoma cell line transplantable in syngenic $\mathrm{WAG} / \mathrm{Rij}$ rats. The cells were cultured in plastic culture flasks $\left(75 \mathrm{~cm}^{2}\right)$ in Roswell Park Memorial Institute Medium 1640 (RPMI 1640) medium supplemented with 20
$\mathrm{mM}$ 4-(2-hydroxyethyl)-1-piperazineethanesulfonic acid (HEPES) buffer solution (Gibco, Invitrogen Ltd., Basel, Switzerland), $50 \mu \mathrm{g} / \mathrm{mL}$ streptomycin and $50 \mathrm{U} / \mathrm{mL}$ penicillin and $10 \%$ foetal calf serum (Amimed, Allschwil, Switzerland) at $37^{\circ} \mathrm{C}$ in $5 \% \mathrm{CO}_{2}$ atmosphere. After 55 passages, in vitro cells were washed with phosphate-buffered saline solution Hank's balanced salt solution (HBSS) (Gibco, Invitrogen Ltd., Basel, Switzerland), trypsinised with Trypsin/diaminoethanetetraacetic acid (EDTA) (Gibco, Invitrogen Ltd., Basel, Switzerland) for $5 \mathrm{~min}$ at $37^{\circ} \mathrm{C}$ and centrifuged for $6 \mathrm{~min}$ at $290 \mathrm{~g}$. After resuspension in RPMI 1640 medium, the number and viability of the tumour cells were measured using the trypan blue method. Viability always exceeded $95 \%$. The cell suspension was diluted using RPMI 1640 medium and fractioned to obtain $200 \mu \mathrm{L}$ samples containing 500,000 CC531s cells each and used for subcapsular hepatic in vivo injection within 15 min of cell harvesting.

\section{Study Design}

Thirty rats were randomised into two surgical groups: $\mathrm{CO}_{2}$ laparoscopy $(n=15)$ as the experimental group and laparotomy $(n=15)$ as the control group. At 10, 15 and 20 days after surgery, the animals were characterised by body weight, liver weight and volume and by the tumour volume by MR imaging, including computed 3D volumetry and peritumoural count of macrophages by local stereology on confocal laser-scanning microscopic images.

\section{Surgical Procedures and Anaesthesia}

The animals were anaesthetised by inhaling a mixture of isoflurane and oxygen $(0.5-5 \%$ isoflurane complemented with pure oxygen) using a conventional vaporiser and a table-fixed animal mask (Provet AG, Lyssach, Switzerland). After induction of narcosis in the induction box, the animals were secured in a supine position on a small-animal operating table and then shaved. The overall operating time was 90 min.

A pneumoperitoneum was provided by a $\mathrm{CO}_{2}$ insufflator (Aesculap, Tuttlingen, Germany). To avoid intra-abdominal pressure fluctuation due to instrument and trocar movements, a 5-L gas tank was placed between insufflator and the abdominal cavity. Laparoscopic imaging was performed by a $30^{\circ} 4 \mathrm{~mm}$ arthroscope with a $4.5 \mathrm{~mm}$ trocar sleeve (Aesculap, Tuttlingen, Germany) and laparoscopic manipulations using two $3 \mathrm{~mm}$ trocars (working channels) and 
$2.7 \mathrm{~mm}$ instruments (scissors, straight forceps; Wolf, Tuttlingen, Germany) introduced under endoscopic guidance. Intra-abdominal hepatic tumour cell inoculation began with the blunt preparation of liver lobes 2 (distal from portal vein) and 5 (proximal to portal vein) using $5 \mathrm{~mm}$ cotton swabs to move the intestines. Three cotton swabs were smoothly placed on the liver and kept ready to stop possible bleeding. A 12-cm-long catheter (needle and adaptor: LUER Lock $0.4 \times 12 \mathrm{~mm}$, silicone tubing $0.6 \mathrm{~mm}$ OD; Ulrich AG, St. Gallen, Switzerland) was introduced to the abdominal cavity by a $2 \mathrm{~mm}$ abdominal wall incision at the right lower abdomen. The incision was clamped to avoid gas leakage. At 45 min after setup, 500,000 CC531s tumour cells in $200 \mu \mathrm{L}$ RPMI 1640 were inoculated by tangential subcapsular ${ }^{15}$ hepatic injection via needle-armed catheter. The catheter was only retracted 5 min later to avoid a backflow of the cell suspension. Finally, all trocar sleeves were removed under endoscopic view to exclude possible bleeding. The wounds then were closed with two absorbable stitches by $3 / 0$ Vicryl ${ }^{\circledR}$.

Open laparotomy as a control was provided by a standardised $5 \mathrm{~cm}$ midline incision and the abdominal wall was retracted using hooks (made of ordinary paperclips) and rubber bands fixed to a metal arch positioned across the rat. Ordinary small-sized hand surgery instruments were used for all open procedures. Tumour cell inoculation was performed applying the same time point and technique as during laparoscopy. The abdominal cavity was protected with moistened sterile gauze during the absence of surgical manipulation. The wound was closed with $3 /$ 0 Vicryl ${ }^{\circledR}$ running sutures in two layers.

\section{Magnetic Resonance Imaging and Computed Three- Dimensional Volumetry}

Following euthanasia, immediate MR tomography was performed in a Somatom Vision (1.5 Tesla, Siemens, Erlangen, Germany) using an MRI wrist coil followed by liver explantation and tissue harvesting. Hepatic tumour nodules in lobes 2 and 5 were detected by postinterventional computed three-dimensional reconstruction and the median tumour volume was determined in $\mathrm{mm}^{3}$.

\section{Tissue Preparation}

MR imaging was followed by immediate liver explantation. The livers were weighed (in grams) and the liver volume was measured by water displacement (in $\mathrm{mm}^{3}$ ). The liver lobes $1-5$ were dissected, washed in phosphate buffered saline (PBS) solution and kept in paraformaldehyde (Fluka, Buchs, Switzerland) until lobes 2 and 5 were processed by the cryostat. Liver cryostat sections of $40 \mu \mathrm{m}$ were air dried for 12 $\mathrm{h}$ and fixed in acetone for $10 \mathrm{~min}$ at room temperature. Standardised immunohistochemical analysis was performed using a conventional staining machine.

\section{Immunohistochemical Analysis}

Primary mouse anti-rat ED 1 monoclonal antibody $(\mathrm{mAb})$ reacting, with a single-chain glycoprotein that is expressed predominantly on the lysosomal membrane of myeloid cells, was used for a general staining of tissue macrophages as well as freshly homed monocytes (Serotec Ltd., Oxford, UK). mAb were tested for their correct staining reaction in a pilot sample and immunohistochemical protocols were adapted to obtain a maximal quality of staining.

Cryostat sections were incubated overnight at $4{ }^{\circ} \mathrm{C}$ with mouse anti-rat ED1 mAb (1:1500, TBS $+0.1 \%$ Triton $+10 \%$ SB; Serotec Ltd., Oxford, UK). After rinsing three times with TBS for $5 \mathrm{~min}$, sections were incubated for $4 \mathrm{~h}$ with goat anti-mouse fluorescein-5isothiocyanate (FITC) (Tris pH 8.2, 1:100; Serotec Ltd., Oxford, UK). After rinsing three times with TBS for $5 \mathrm{~min}$, sections were developed by incubating for $10 \mathrm{~min}$ with propidiumiodide $(1 \mathrm{mg} / 100 \mathrm{ml} \mathrm{PBS}$ pH 7.3; Serotec Ltd., Oxford, UK).

After rinsing with TBS ( $\mathrm{pH}$ 7.3) for $10 \mathrm{~min}$, sections were mounted with Slow Fade ${ }^{\circledR}$ (Molecular Probes, Leiden, The Netherlands).

\section{Confocal Microscopy and Local Stereology for Peritumoural Macrophage Count}

A transtumoural $40 \mu \mathrm{m}$ cryosection in both lobes was sampled at the each tumour's largest diameter and analysed by a confocal laser-scanning microscope (Eclipse 800, Nikon, Germany/Bio Rad Microscopy Division, Herts, UK) after staining. Macrophages were quantified within five peritumoural and five parenchymateous counting frames over five optical plains with an in-between distance of $2 \mu \mathrm{m}$ (volume of assessed cubes: $80 \times 80 \times 10 \mu \mathrm{m})$. These frames were sampled as shown in Fig. 1, Fig. 2, Fig. 3, Fig. 4: a basic axis through a random point within the tumour nodule and orthogonal to the liver's surface was selected. Furthermore, at $\pm 30^{\circ}$ and $\pm 60^{\circ}$, additional axes were selected running through the same random point. At the cross section of these axes with the 

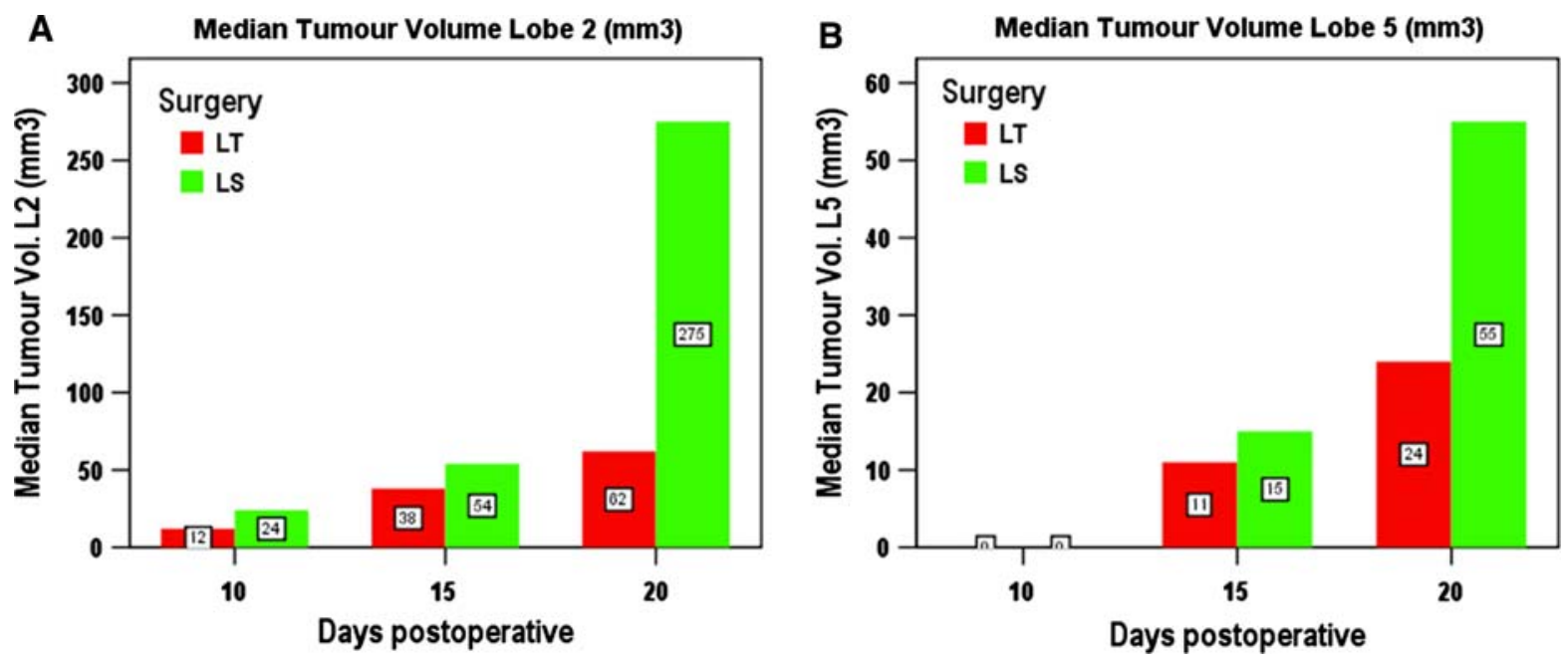

FIG. 1. Median tumour volumes of hepatic colorectal adenocarcinoma nodule in liver lobe 2 (Fig. 1A) and lobe 5 (Fig. $1 \mathrm{~B}$ ) in $\mathrm{mm}^{3}$ at 10,15 and 20 days after $\mathrm{CO}_{2}$ laparoscopy (green) and laparotomy (control, red) measured by immediate post-mortem MR imaging and consecutive computed three-dimensional reconstruction and volumetry $(n=5) ; \mathrm{p}<0.05$ for differences between the surgical groups at any time point in lobe 2, and for 15 and 20 days after laparoscopy in lobe 5 (Mann-Whitney test).
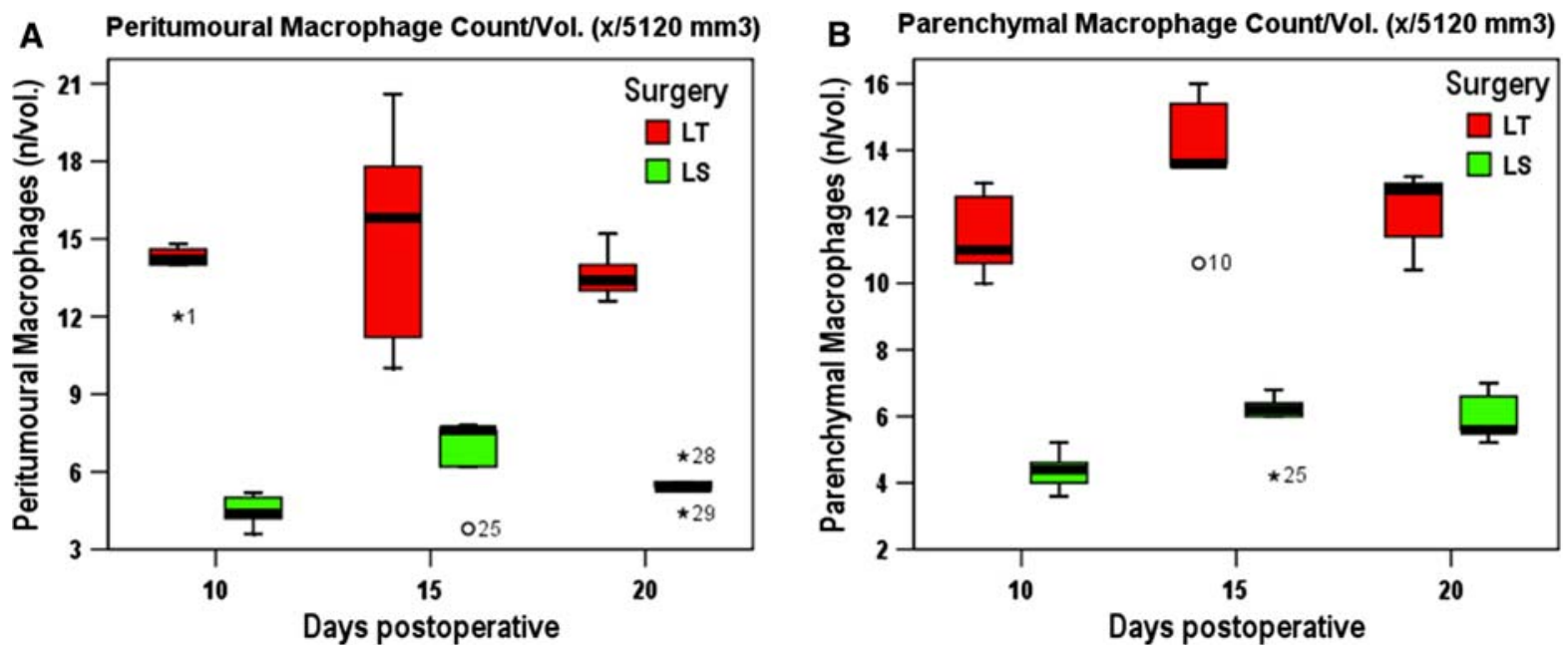

FIG. 2. Median number of hepatic peritumoural (A) and parenchymal (B) macrophages within liver lobe 2 in count $/ 5120 \mathrm{~mm}^{3} 10,15$ and 20 days after $\mathrm{CO}_{2}$ laparoscopy (green) and laparotomy (control, red) measured by specific immunohistological staining and morphometric analysis using confocal fluorescent laser-scanning microscopy $(n=5)$; $\mathrm{p}$ values $<0.05$ for differences between laparoscopy and laparotomy at any time point (Mann-Whitney test).

tumour borders, the five peritumoural counting fields and at a radial distance of $160 \mu \mathrm{m}$ from the tumour's margin, five parenchymateous counting fields were defined (see Fig. 4). The median number of macrophages within the peritumoural and parenchymateous volumes was analysed. Importantly, only the overlay of a propidiumiodide-stained nucleus with the ED1-stained cytoplasma was considered to be a macrophage and omitted in subsequent planes to avoid bias.

\section{Statistical Methods}

Liver weights and volumes are presented as mean \pm standard deviation (SD). Means between the two groups (laparoscopy versus laparotomy) were compared by Student's $t$-test after having tested for normal distribution.

Tumour volumes and macrophage counts are given as median values. Significance testing between the two groups was performed by the Mann-Whitney test. Analysis of tumour volumes between the two lobes (2 

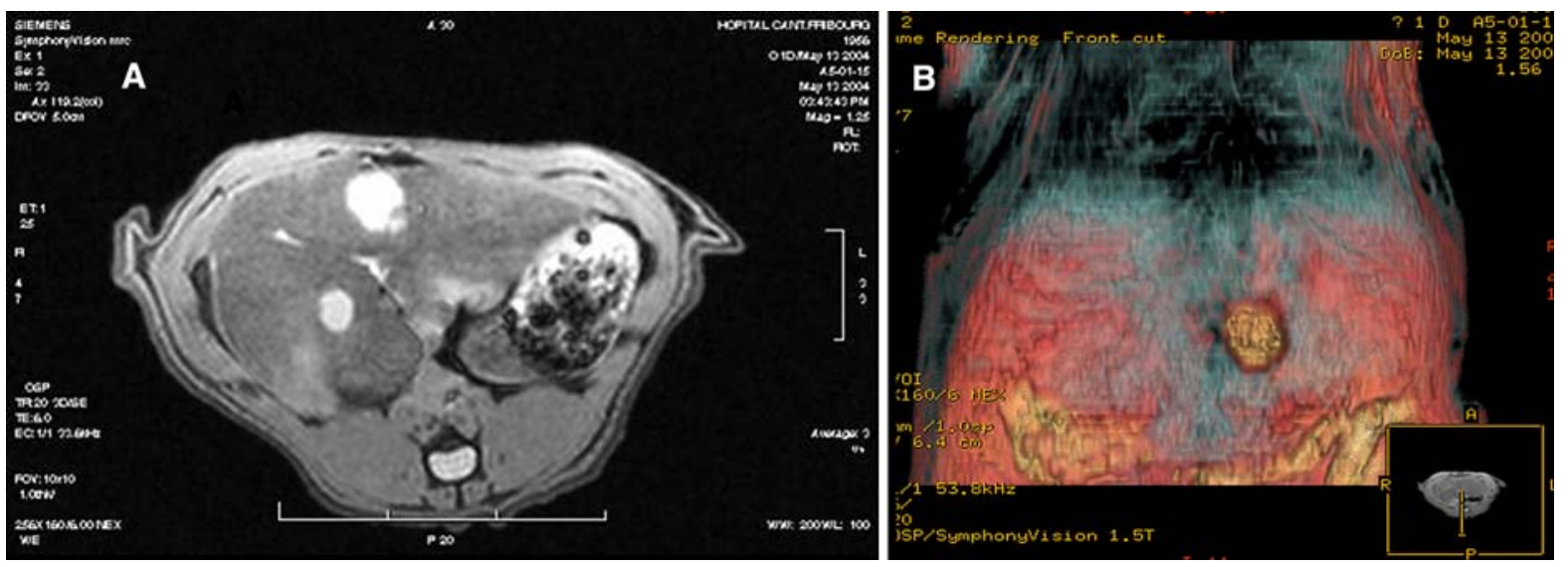

FIG. 3. Conventional and 3D-MR imaging of hepatic colorectal adenocarcinoma metastasis: (A) immediate post-mortem conventional MR imaging and (B) three-dimensional computed reconstruction of hepatic colorectal adenocarcinoma metastasis of the CC531s cell line in WAG/ $\mathrm{Rij}$ rats after subcapsular cellular injection during $\mathrm{CO}_{2}$ laparoscopy at $12 \mathrm{mmHg}$ intra-abdominal pressure 20 days before imaging.
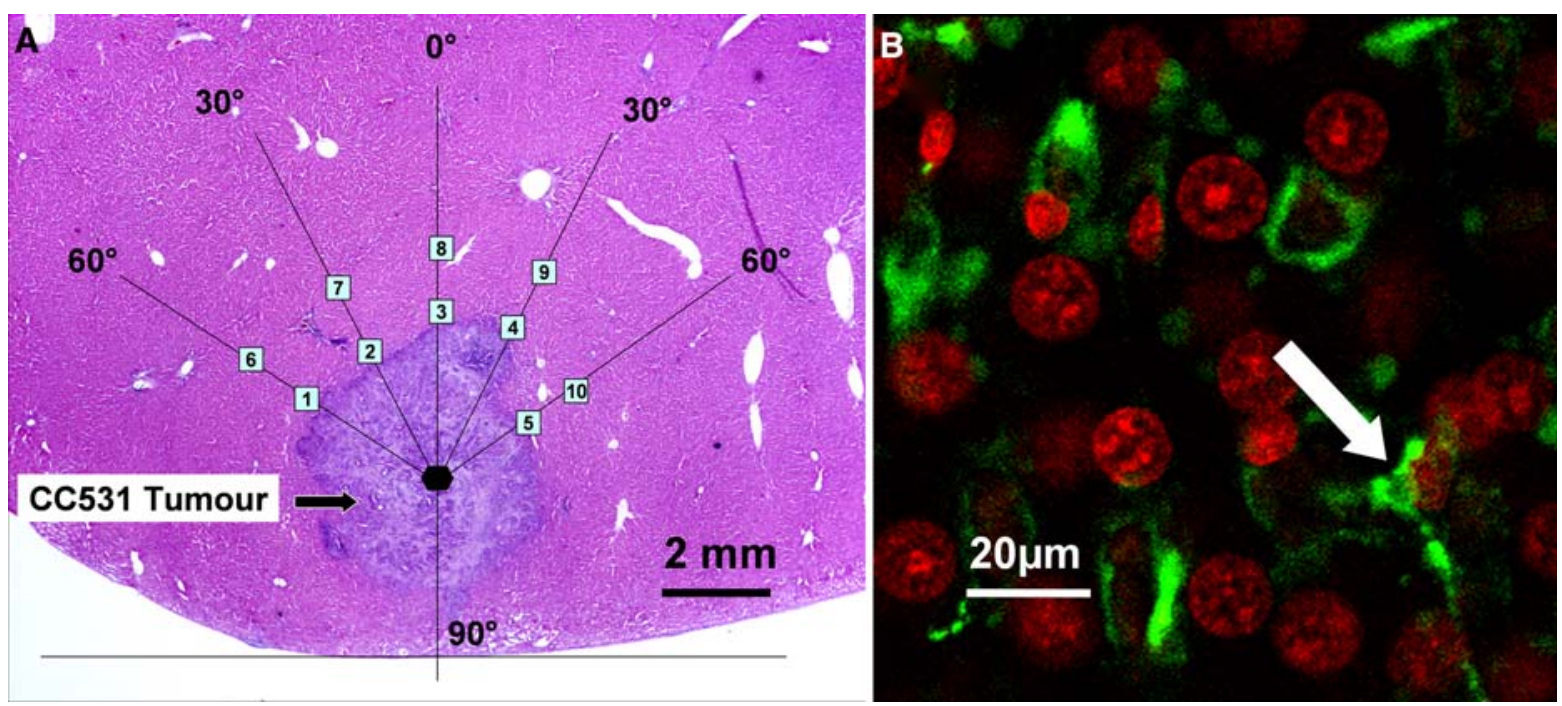

FIG. 4. Histological sampling for confocal laser scanning morphometry. (A) Histological haematoxylin-eosin-stained section of a hepatic CC531s colon adenocarcinoma metastasis $(\Leftrightarrow)$ and schematic counting frames for morphometric analysis within peritumoural (1-5) and parenchymal (6-10) counting fields. (B) Fluorescent immunohistological staining for macrophages (primary antibody ED1; Serotec/propidiumiodide) in a counting frame within a counting region for three-dimensional quantification of macrophages $(\Leftrightarrow)$ over a depth of $10 \mu \mathrm{m}$ by fluorescent confocal laser-scanning microscope.

and 5) as well as the macrophage counts between the peritumoural and parenchymal areas at a certain time point were provided by an unpaired $t$-test.

Significance was accepted in all cases at $p<0.05$. All statistical procedures were performed using SPSS ${ }^{\circledR}$ for Windows (SPSS Science International, Chicago, Illinois, USA).

\section{RESULTS}

The mean body weight of the rats at the time point of surgery was $268 \pm 37.6 \mathrm{~g}$ in animals undergoing laparotomy and $281 \pm 27.9 \mathrm{~g}$ in the laparoscopic group. There was no significant difference between the surgical groups $(p>1)$.

The mean total liver weights ranged from $11.4 \pm 2.1 \mathrm{~g}$ to $14.1 \pm 1 \mathrm{~g}$, the mean total liver volumes ranged from $10.5 \pm 2 \mathrm{~cm}^{3}$ to $13.1 \pm 0.9$ $\mathrm{cm}^{3}$, the mean volume of the liver's second lobe ranged from $3.7 \pm 0.6 \mathrm{~cm}^{3}$ to $4.7 \pm 0.3 \mathrm{~cm}^{3}$ and the mean volume of the fifth liver lobe ranged from $0.4 \pm 0.2 \mathrm{~cm}^{3}$ to $0.7 \pm 0.2 \mathrm{~cm}^{3}$ (see Table 1). However, there were no significant differences for these values between the surgical groups at any postoperative time point $(p>1)$. 
TABLE 1. Mean total liver weight, total liver volume and volume of hepatic lobes 2 and 5

\begin{tabular}{lccc}
\hline & 10 days post-op. & 15 days post-op. & 20 days post-op. \\
\hline Total liver weight $(\mathrm{g})$ & & & $13.16 \pm 1.16$ \\
Laparotomy & $11.42 \pm 2.1$ & $12.44 \pm 1.38$ & $14.14 \pm 0.99$ \\
$\begin{array}{l}\text { Laparoscopy } \\
\text { Total liver volume }\left(\mathrm{cm}^{3}\right)\end{array}$ & $12.04 \pm 0.71$ & $13.04 \pm 1.15$ & $12.16 \pm 1.13$ \\
Laparotomy & $10.52 \pm 2.02$ & $11.58 \pm 1.41$ & $13.10 \pm 0.92$ \\
Laparoscopy & $12.18 \pm 0.61$ & $12.85 \pm 1.08$ & $4.3 \pm 0.5$ \\
Volume of hepatic lobe $2\left(\mathrm{~mm}^{3}\right)$ & $3.7 \pm 0.6$ & $4.1 \pm 0.4$ & $4.7 \pm 0.3$ \\
Laparotomy & $4.1 \pm 0.5$ & $4.4 \pm 0.4$ & $0.7 \pm 0.1$ \\
Laparoscopy & $0.4 \pm 0.2$ & $0.7 \pm 0.1$ & $0.7 \pm 0.2$ \\
Volume of hepatic lobe $5\left(\mathrm{~mm}^{3}\right)$ & $0.7 \pm 0.7$ & $0.7 \pm 0.1$ & 0.7 \\
Laparotomy & Laparoscopy & &
\end{tabular}

There was no significant difference in mean total liver weight, total liver volume or lobal volume 2 or 5 between the two groups at any time point after surgery ( $p>1$; Student's $t$-test after testing for normal distribution).

The median nodular volume of colorectal adenocarcinoma metastases after $\mathrm{CO}_{2}$ laparoscopy was significantly higher than after laparotomy at any measured time point following surgery in hepatic lobe 2 and at 15 and 20 days after surgery in hepatic lobe 5 $(p<0.01 ;$ Mann-Whitney test). All animals of either group developed tumours in lobe 2 and all animals of either group sacrificed at 15 and 20 days after surgery developed tumours in lobe 5. However at 10 days after surgery, in only one of five animals of either group was a tumour nodule detected in lobe 5 by both MRI and histological assessment (volume after LT $7 \mathrm{~mm}^{3}$; after LS $15 \mathrm{~mm}^{3}$ ). 20 days after surgery, the tumour volume after laparoscopy was four times higher in lobe 2 and more than double in lobe 5 compared to laparotomy. Furthermore, the tumour volumes were significantly higher in lobe 2 compared to lobe 5 in both groups at any time point $(p<0.05$, unpaired $t$-test).

The median number of hepatic macrophages in lobe 2 within the peritumoural as well as the parenchymal counting fields was significantly lower after $\mathrm{CO}_{2}$ laparoscopy than after laparotomy at all time points after surgery $(p<0.01$, Mann-Whitney test). Histological quantification of macrophages in lobe 5 was omitted due to a lack of stereological reference due to undetectable tumour nodules 10 days after surgery. No significant difference between peritumoural and parenchymateous counting was detected $(p<0.05$, unpaired $t$-test $)$.

\section{DISCUSSION}

In the present study, the overall postoperative tumour growth of hepatic metastasis of colorectal adenocarcinoma after experimental laparoscopy in rats was significantly increased and associated with a lower peritumoural macrophage count than after laparotomy up to 20 days after surgery, with a lack of significant difference in preoperative bodyweight or postoperative liver weight and volume at the time point of MRI and liver explantation between the surgical groups.

To imitate abdominal surgery (laparoscopy/laparotomy) in patients suffering from colonic or rectal adenocarcinoma with hepatic metastases, preference was given to the subcapsular hepatic injection of CC531s colon adenocarcinoma cells. Previous applications of the CC531s model in experimental laparoscopy were performed by intrasplenic ${ }^{7}$ or intraportal $^{8,9}$ injection of the tumour cells, leading to multiple hepatic metastases after haematogeneous spread. In contrast, CC531s cells were injected subcapsularly in a nonsurgical oncological study to induce well-localised and accessible liver metastases. ${ }^{16}$ However, the present study combines the two techniques in order to avoid multiple haematogeneous hepatic tumour seeding and to focus on the postoperative development of single, well-localised hepatic colorectal metastases. Technically, the model of experimental laparoscopy previously described ${ }^{17,18}$ was slightly modified: intra-abdominal pressure fluctuations during laparoscopy were equilibrated by a 5$\mathrm{L}$ gas tank between the inflator and the abdominal cavity in accordance with the average human intraabdominal volume and the performance of subcapsular injection by a flexible silicone catheter with a metallic needle point instead of a syringe with a rigid needle-top provoking a pressure on the inflated abdomen $^{7,8}$ in the moment of tumour cell inoculation. Previous semiquantitative tumour measures (Steller Score, cancer index ${ }^{7}$ or metastatic index ${ }^{18,19}$ ) were replaced by quantitative volumetry by MR imaging. 
Regarding pathophysiological and tumour biological aspects, the method of subcapsular hepatic tumour cell injection is highly debatable: in the event of open or laparoscopic resection of colorectal cancer and the postoperative formation of hepatic metastases eventual pre- or perioperative tumour cell seeding most probably occurs mainly due to haematogeneous or lymphogeneous spreading. This aspect is completely lost by injecting tumour cells subcapsularly and directly into the hepatic tissue. Subcapsular inoculation furthermore confronts the liver with a localised exorbitantly high load of viable tumour cells, which probably would never reach peripheral hepatic areas in such high concentrations after haematogeneous spreading, omitting the process of haematogeneous tumour cell spillage with distribution throughout the whole liver and the event of diapedesis (extra-vascularisation of cells). Therefore, previously described models with intraportal ${ }^{8,9}$ or intrasplenic ${ }^{7}$ injection followed by haematogeneous spread appear to represent the pathophysiological mechanism of colorectal tumour cell spillage more reliably. On the other hand, the subcapsular model might serve to imitate the fact of pre-existing or dormant micrometastases at the tim-point of surgery and to observe their postoperative development after open or laparoscopic surgery (e.g. colon resection). Unfortunately, this study's model applies the determining surgical technique (laparoscopy versus laparotomy) synchronically to the tumour cell injection. Hence the tumour nodules cannot be present and established at the time point of variable surgical application and remain difficult to be considered as pre-existing or dormant metastases. Furthermore, interpreting the impact of a surgical method on a metastasis that effectively is not yet established at the time point of surgery is debatable and rather vague. Ideally, the injection and formation of metastases should take place before surgery, as provided by Schmeding et al. ${ }^{20}$ Another source of error may be the quality of inoculation, depending on the technique of injection. If the needle is retracted too early an inevitable reflux of tumour cells impairs the quality of inoculation by a decreased number of cells injected. Finally, it remains uncertain whether a 90 min exposition to laparoscopic or open surgery might result in a significantly different influence on tumour's biology with an impact on postoperative tumour growth up to 20 days after surgery.

The postoperative development of hepatic metastases within the present study showed an overall tendency of significantly increased growth after laparoscopy compared to laparotomy up to 20 days after surgery and significantly higher tumour growth in lobe 2 compared to lobe 5 . However, in lobe 5 after 10 days four of five animals in either surgical group failed to develop any MR or histologically detectable tumour nodule in contrast to all other animals with well-established postoperative tumours in both lobes. Apparently, the quality of injected cells, although tested for viability, or of the injection technique into lobe 5 might not have been to the highest standards, although the corresponding animals displayed welldeveloped tumours in lobe 2. This unexpected lack of tumour growth most probably occurred for a technical reason and indicates how delicate and fragile the model appears to be and as a consequence how difficult it remains to exclude uneven tumour growth due to failed or incomplete cellular implantation. Nevertheless, in the following sections the tendency of an overall increased tumour growth after laparoscopy is considered justified.

The most comparable experimental study was provided by Gutt et al. ${ }^{8}$ by intraportal injection of CC531s adenocarcinoma cells during $\mathrm{CO}_{2}$ laparoscopy and laparotomy, which resulted in no significant difference in postoperative tumour growth between the surgical groups in contrast to this study's findings of increased growth after laparoscopy. Possibly, the induction of two single metastases compared to the affection of the entire liver in Gutt's study is related to a sensitivity to the surgical technique which is absent at a certain tumour load and amount of disease. In this case the more determining factor seems to be the number of tumours instead of the surgical technique, and the situation of single metastases may differ completely from the situation of multilobar hepatic tumour spread. Else, in Gutt's study, the use of semiquantitative macroscopic and microscopic analysis of the tumour load omitting intraparenchymal lesions and applying nonstereological morphometry might also be a source of error. However, the subcapsular technique also has a certain potential of errors due to inefficient inoculation and the absence of haematogeneous intrahepatic distribution of the tumour cells, as demonstrated by the unexpected failure of tumour growth in lobe 5 at 10 days after surgery in eight animals. In another study, Gutt et al. $^{7}$ induced abdominal tumours by cell injection into the spleen. No significant difference in tumour load was found between laparotomy and $\mathrm{CO}_{2}$ laparoscopy in the liver. Intrasplenic injection resulted in haematogeneous tumour cell spillage through the splenic and portal vein, leading to multiple hepatic metastases as seen in the study before. ${ }^{7}$ Apparently, the same difficulties of comparison arise as the situation 
of total hepatic cancerous affection seems to differ from the state of solitary metastases, and semiquantitative measures are unequal to the quantitative stereological measures of the present study. A favourable oncological outcome after $\mathrm{CO}_{2}$ laparoscopy was detected by Bouvy et al. ${ }^{10}$ and Lee et al. ${ }^{11}$ Bouvy administered the tumour cells both into the abdominal cavity and under the renal capsule, omitting the haematogeneous factor and focusing on a well-localisable subcapsular renal tumour nodule. Interestingly, subcapsular injection and consecutive elevated intra-abdominal pressures resulted in less severe tumour growth than did open surgery, although Bouvy used the kidney instead of the liver and thus focused on a retroperitoneal site. However, this model might be of importance when it comes to metastases per continuitatem with direct retroperitoneal invasion of the primary colon tumour. Further immunological assessments were not provided and therefore the influence of the renal tissue and immunological factors remain unclear. At any rate, this model demonstrates the potential aggression of CC531s cells even in nonhepatic tissue and confirms the potential of these cells for malignant inoculation after subcapsular interstitial administration. As the present study intends to imitate the clinical situation of laparoscopy in manifest or dormant hepatic colorectal metastases, despite the ambivalence of synchronic injection and pneumoperitoneum, a trial assessing the influence of laparoscopy on pre-existing metastases would be of great interest. The present state of literature does not reveal any satisfactory study other than a publication by Schmeding et al. ${ }^{20}$ on tumour recurrence after curative partial liver resection in localised, induced hepatocellular carcinoma. This is the only study documenting the influence of laparoscopy on pre-existing hepatic tumour in an experimental model. Schmeding did not detect any significant difference in tumour recurrence at the hepatic site of resection between laparotomy or $\mathrm{CO}_{2}$ laparoscopy. On the contrary, he found helium laparoscopy to be the most protective technique with the least local tumour recurrence after curative resection. Although Schmeding's tumour cell line, injection and surgical techniques and animal model differ from the present study, it remains of great interest regarding the impact of laparoscopy on pre-existing hepatic tumours.

Macrophages play a key role in cellular antitumoural immunological defence in the early onset of hepatic colorectal carcinoma metastases, as shown by selective depletion of macrophages (including Kupffer cells in the liver) associated with an increased hepatic growth of colorectal metastases ${ }^{12,13}$ and activation of macrophages leading to enhanced tumour growth. ${ }^{21}$ The cellular interaction between colonic tumour cells and hepatic macrophages with the CC531s tumour cell line was confirmed by Timmers et al. using immunofluorescent microscopy in an early tumoural onset study. ${ }^{22}$ Timmers et al. applied an intravenous cell inoculation with haematogeneous tumour spillage, limiting their experiments to the first $24 \mathrm{~h}$ after injection, and did not include any laparoscopic approach. These points differ completely from the design of the present study and do not facilitate comparison of the cellular immunological response. While Timmers et al. concentrated on cellular interaction and phagocytotic function rather than numbers of macrophages, Heuff et al. ${ }^{12}$ as well as Bayon et al. ${ }^{13}$ defined phagocytotic populations and performed semiquantitative measures in purely oncological experimental studies. Heuff et al., ${ }^{12}$ besides enhanced tumour growth after selective macrophage depletion, found a marked increase in the total macrophage population as well as the tissue-resident subpopulation in the liver parenchyma 2 weeks after tumour injection. As a consequence, the number of freshly homed macrophages was also elevated. These immunological cells were mainly located in the portal and central areas. There was no peritumoural assessment. Bayon et al. ${ }^{13}$ reported the same findings with over $75 \%$ of freshly homed macrophages among the macrophages with intratumoural localisation at 2 as well as 14 days after inoculation. No peritumoural inflammatory corona was detected and no parenchymal macrophage assessment in the normal liver tissue was provided. Regarding the high impact of freshly homed macrophages on antitumoural immunological defence up to 2 weeks after injection compared to the population of tissue resident macrophages (Kupffer cells) disclosed in the Bayon and Heuff studies, only general macrophage staining was provided in the present study. Although the other two studies mentioned do not refer to any laparoscopic technique, an enhanced tumour growth after laparoscopy might be explained by associated decreased numbers of peritumoural and parenchymal macrophages. Importantly, Gutt et al. ${ }^{8}$ in their semiquantitative analysis of hepatic colorectal tumour growth and accompanying Kupffer cells after laparoscopy found no difference in Kupffer cell count at an indifferent tumour load after laparoscopy and laparotomy. These findings differ from the increased tumour volumes and lower macrophage counts after laparoscopy in the present study. This discrepancy might again occur due to haematogeneous tumour 
cell spillage into all liver segments causing a completely different immunological situation within the hepatic tissue. The measurement took place 28 days after surgery, whereas the latest time point of measurement in the present study was at 20 days after surgery only. Possibly, a direct influence of laparoscopy on tissue resident macrophages might have been equilibrated by 28 days after surgery. Methodologically, nonstereological semiquantitative macrophage analysis might be another reason of discrepant results, or the semiquantitative measures were provided by staining of tissue-resident macrophages (Kupffer cells) omitting freshly homed macrophages. This might explain why the macrophage count of the present study, including all kinds of macrophages (tissue-resident and freshly homed), after laparoscopy turns out to be higher than after laparotomy. However, as Bayon et al. ${ }^{13}$ documented over $75 \%$ freshly homed macrophages, despite a mostly intratumoural measurement, this subpopulation appears to play an important role in antitumoural cellular defence. Furthermore, to date no quantitative differentiation between macrophage count directly attached to the tumour and within the remnant parenchymal liver tissue has been performed and the absence of a significant difference in the present study implies the absence of an overshooting immunological corona around the tumour, which is perfectly in accordance with the findings of Bayon et al. 14 days after tumour injection. ${ }^{13}$ The absence of a significant difference in macrophage numbers around the tumour compared to the remnant liver tissue might indicate a generally modified overall hepatic antitumoural cellular response after laparoscopy compared to laparotomy instead of a local immunological influence. As previous studies could not distinguish any postoperative difference in tumour load nor macrophage counts after laparoscopy and laparotomy, ${ }^{8}$ the impact of laparoscopy and $\mathrm{CO}_{2}$ detected in the present study might be irrelevant for larger amounts of disease all over the liver, although an impaired immunological reaction would neatly explain a greater tumour load.

In conclusion, MR tumour volumetry and stereological immunological analysis in an animal model resulted in an increased overall postoperative tumour growth associated with decreased numbers of hepatic macrophages after experimental $\mathrm{CO}_{2}$ laparoscopy. Although previous studies could not distinguish any difference in tumour growth and antitumoural cellular defence after haematogeneous tumour cell spillage over the entire liver and though the influence of $90 \mathrm{~min}$ of laparoscopy on tumour growth and immunological response up to 20 days postopera- tively is debatable, the present significant postoperative unfavourable oncological tendency in solitary metastases questions the safety of experimental laparoscopy in manifest or potential hepatic metastases. Further experimental studies on the regulation of the cellular immunological and tumoural hepatic reaction as well as clinical studies are needed to answer the question of whether to provide laparoscopy or open surgery in patients with hepatic colorectal metastases.

\section{ACKNOWLEDGEMENTS}

The authors would like to thank the Swiss National Foundation (Grant No. 3200-061486.00) and the Swiss Foundation for Molecular Tumour Research for their great financial support. Furthermore, we highly acknowledge the technical support by BBraun Aesculap Switzerland, Ethicon Switzerland, Tyco Healthcare Switzerland. We address many thanks to Prof Marco Celio, Head of the Department of Medicine, University of Fribourg for his laboratory support and Brigitte Belser and Valerie Salicio and their teams for the work in the histological and cell-culture laboratory.

\section{REFERENCES}

1. Martin LC, Puente I, Sosa JL, et al. Open versus laparoscopic appendectomy. A prospective randomized comparison. Ann Surg 1995; 222:256-61.

2. McMahon AJ, Russell IT, Baxter JN, et al. Laparoscopic versus minilaparotomy cholecystectomy: a randomised trial. Lancet 1994; 343:135-8.

3. Steiner CA, Bass EB, Talamini MA, et al. Surgical rates and operative mortality for open and laparoscopic cholecystectomy in Maryland. N Engl J Med 1994; 330:403-8.

4. A comparison of laparoscopically assisted and open colectomy for colon cancer. N Engl J Med 2004; 350:2050-9.

5. Guillou PJ, Quirke P, Thorpe H, et al. Short-term endpoints of conventional versus laparoscopic-assisted surgery in patients with colorectal cancer (MRC CLASICC trial): multicentre, randomised controlled trial. Lancet 2005; 365:1718-26.

6. Veldkamp R, Kuhry E, Hop WC, et al. Laparoscopic surgery versus open surgery for colon cancer: short-term outcomes of a randomised trial. Lancet Oncol 2005; 6:477-84.

7. Gutt CN, Riemer V, Kim ZG, et al. Impact of laparoscopic surgery on experimental hepatic metastases. Br J Surg 2001; 88:371-5.

8. Gutt CN, Kim ZG, Schemmer P, et al. Impact of laparoscopic and conventional surgery on Kupffer cells, tumor-associated CD44 expression, and intrahepatic tumor spread. Arch Surg 2002; 137:1408-12.

9. Ishida H, Hashimoto D, Takeuchi I, et al. Liver metastases are less established after gasless laparoscopy than after carbon dioxide pneumoperitoneum and laparotomy in a mouse model. Surg Endosc 2002; 16:193-6.

10. Bouvy ND, Marquet RL, Jeekel J, et al. Laparoscopic surgery is associated with less tumour growth stimulation than con- 
ventional surgery: an experimental study. Br J Surg 1997; 84:358-61.

11. Lee SW, Gleason N, Blanco I, et al. Higher colon cancer tumor proliferative index and lower tumor cell death rate in mice undergoing laparotomy versus insufflation. Surg Endosc 2002; 16:36-9.

12. Heuff G, Oldenburg HS, Boutkan H, et al. Enhanced tumour growth in the rat liver after selective elimination of Kupffer cells. Cancer Immunol Immunother 1993; 37:125-30.

13. Bayon LG, Izquierdo MA, Sirovich I, et al. Role of Kupffer cells in arresting circulating tumor cells and controlling metastatic growth in the liver. Hepatology 1996; 23:1224-31.

14. Gutt CN, Heinz P, Kaps W, et al. The phagocytosis activity during conventional and laparoscopic operations in the rat. A preliminary study. Surg Endosc 1997; 11:899-901.

15. Isbert C, Boerner A, Ritz JP, et al. In situ ablation of experimental liver metastases delays and reduces residual intrahepatic tumour growth and peritoneal tumour spread compared with hepatic resection. Br J Surg 2002; 89:1252-9.

16. Hagenaars M, Ensink NG, Basse PH, et al. The microscopic anatomy of experimental rat CC531 colon tumour metastases: consequences for immunotherapy? Clin Exp Metastasis 2000; 18:189-96.

17. Gutt CN, Riemer V, Brier C, et al. Standardized technique of laparoscopic surgery in the rat. Dig Surg 1998; 15:135-9.

18. Gutt CN, Kim ZG, Gessmann T, et al. Hepatic tumor spread of colorectal cancer in a laparoscopic animal model. Surg Endosc 2000; 14:448-51.

19. Ku Y, Kusunoki N, Shiotani M, et al. Stimulation of haematogenous liver metastases by ischaemia-reperfusion in rats. Eur J Surg 1999; 165:801-7.

20. Schmeding M, Schwalbach P, Reinshagen S, et al. Helium pneumoperitoneum reduces tumor recurrence after curative laparoscopic liver resection in rats in a tumor-bearing small animal model. Surg Endosc 2003; 17:951-9.

21. Williams DL, Sherwood ER, McNamee RB, et al. Therapeutic efficacy of glucan in a murine model of hepatic metastatic disease. Hepatology 1985; 5:198-206.

22. Timmers M, Vekemans K, Vermijlen D, et al. Interactions between rat colon carcinoma cells and Kupffer cells during the onset of hepatic metastasis. Int J Cancer 2004; 112:793-802. 